\title{
Incidence of Hepatocellular Carcinoma Reduced by Phlebotomy Treatment in Patients with Chronic Hepatitis C
}

\author{
Kazushige Nirei, Shuniti Matsuoka, Hitomi Nakamura, \\ Hiroshi Matsumura and Mitsuhiko Moriyama
}

\begin{abstract}
Objective To determine the impact of phlebotomy on the laboratory values and the incidence of hepatocellular carcinoma (HCC) in patients with hepatitis C.

Methods Study patients with chronic hepatitis $\mathrm{C}$ were treated with glycyrrhizin injection and oral ursodeoxycholic acid and either with $(n=52)$ or without $(n=50)$ phlebotomy during the period of 2005-2012. Six phlebotomized patients had previously received interferon therapy and were subsequently excluded from the data analysis. The serum levels of aspartate aminotransferase (AST), alanine aminotransferase (ALT), total bilirubin, alkaline phosphatase, gamma-glutamyl transpeptidase, lactate dehydrogenase, ferritin, iron and albumin, as well as the hemoglobin concentration, platelet count and prothrombin time, were determined. We compared the long-term outcomes based on the incidence of HCC and laboratory values, including the baseline serum ferritin levels, in patients treated with versus without phlebotomy.

Results In the phlebotomy group, the mean AST and ALT levels decreased significantly at each one-year interval over five years $(\mathrm{p}<0.01)$, whereas the platelet counts did not. The incidence of HCC in the phlebotomized patients was significantly lower than that observed in the patients treated without phlebotomy: $10.3 \%$ vs. $43.7 \%$, respectively, during the 8 -year observation period ( $\mathrm{p}=0.04$ ). The incidence of $\mathrm{HCC}$ was also lower in the phlebotomized patients with a normal baseline ferritin level: $0.0 \%$ vs. $36.0 \%$ in the matched subgroup treated without phlebotomy at year 8 . Phlebotomy offered a risk ratio of 0.13 , thus suggesting protection against the development of HCC.

Conclusion The incidence of HCC can be reduced by phlebotomy treatment, which should be performed in patients with chronic hepatitis $\mathrm{C}$ not receiving or not responding to antiviral therapy.
\end{abstract}

Key words: carcinogenesis, hepatitis C, phlebotomy, iron overload, hepatocellular carcinoma

(Intern Med 54: 107-117, 2015)

(DOI: 10.2169/internalmedicine.54.2715)

\section{Introduction}

The use of pegylated interferon (IFN) and ribavirin combination therapy in patients infected with the hepatitis $\mathrm{C}$ virus (HCV) achieves clearance of $\mathrm{HCV}$ in approximately $50 \%$ of cases, even those involving genotype $1 \mathrm{~b} \mathrm{HCV}$ infection and a high viral load. Furthermore, with this combination treatment in addition to protease inhibitors, it has become possible to achieve sustained virologic response rates approaching 70\% in Japan (1-3).

However, significantly reduced rates of a sustained virologic response are observed in women, cases involving a reduced dose or discontinuation of both IFN and ribavirin and patients with substitutions of amino acid 70 and/or 91 in the HCV core region (4), mutations in the gene encoding nonstructural protein 5A (5) or genetic polymorphisms near the $I L 28 B$ gene $(6,7)$. In such cases, it is necessary to maintain lower levels of aspartate aminotransferase (AST) and alanine aminotransferase (ALT) with glycyrrhizin injection 


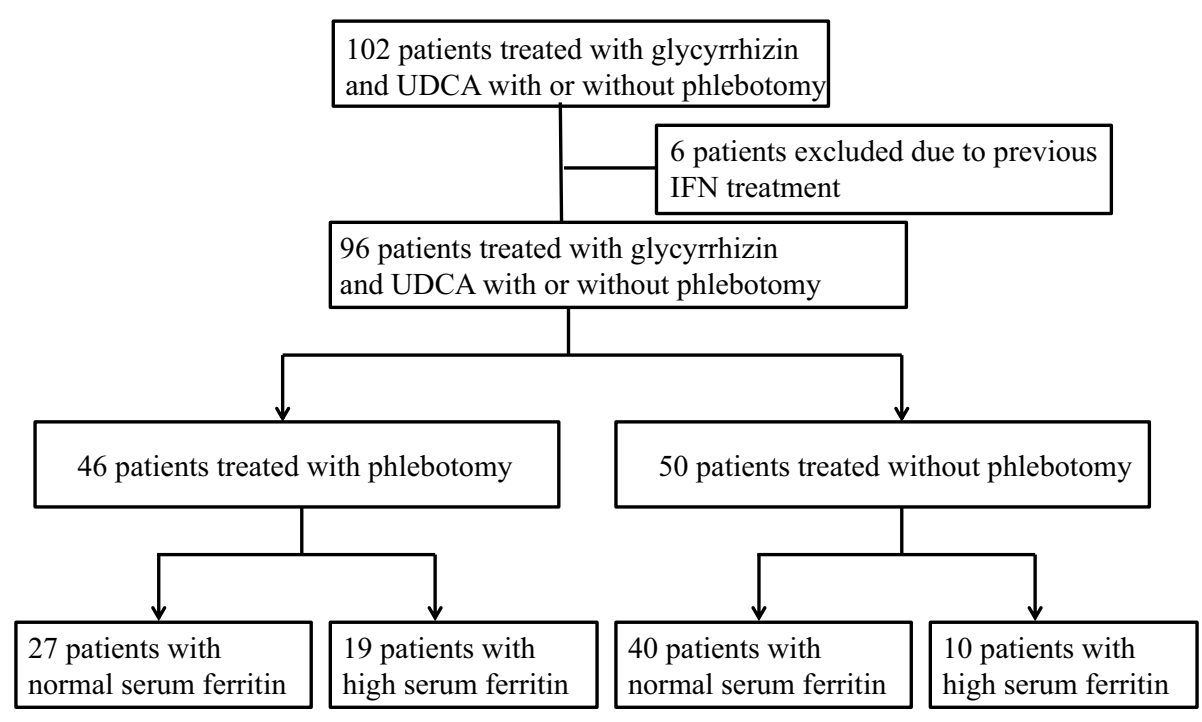

Figure 1. Patients treated with glycyrrhizin injection and oral UDCA in the phlebotomy and nonphlebotomy groups.

and oral ursodeoxycholic acid (UDCA) therapy, which reduces the risk of occurrence of hepatocellular carcinoma (HCC) resulting from chronic hepatitis $\mathrm{C}$ and can significantly improve the quality of life (8-11). It has recently been reported that risk factors for HCC include iron overload (12). Fortunately, the risk of carcinogenesis and liver dysfunction can be suppressed with iron reduction therapy, including phlebotomy (13).

In the present report, we conducted a cohort study comparing the long-term outcomes in two treatment groups of patients with chronic hepatitis $\mathrm{C}$ who underwent treatment with glycyrrhizin injection and oral UDCA with or without phlebotomy. Our study aims were to determine the impact of phlebotomy on the serum laboratory values and incidence of HCC as well as examine the influence of the baseline ferritin level.

\section{Materials and Methods}

\section{Study population}

This study was performed prospectively. Patients with chronic hepatitis $\mathrm{C}$ participated in the study performed at Nihon University School of Medicine Hospital (Tokyo, Japan) from January 2005 through December 31, 2012. All patients provided their informed consent to participate in the study. The study protocol specified that no patients should have previously received IFN therapy. Similarly, if a patient started IFN therapy, we discontinued their follow-up in the study. All study patients were treated with glycyrrhizin injection and oral UDCA administration, and comparisons were made between those additionally treated with and without phlebotomy. A comparison was also made between patients whose baseline serum ferritin level was within the normal range versus those with an increased baseline serum ferritin level. The upper limit of a normal ferritin level was defined as $200 \mu \mathrm{g} / \mathrm{L}$ for women and $300 \mu \mathrm{g} / \mathrm{L}$ for men, as per the Hemochromatosis and Iron Overload Screening Study (14). All patients tested positive for serum HCV RNA (measured using the Amplicor HCV Monitor; Roche Diagnostics K.K., Tokyo, Japan) and negative for serum hepatitis B surface antigens (measured using an enzyme-linked immunosorbent assay; Dinabot, Tokyo, Japan). No heavy drinkers (defined as an ethanol intake of $>30 \mathrm{~g}$ per day) were included in the study.

Patients with Child class $\mathrm{C}$ cirrhosis, anemia (defined as a hemoglobin level of $<11 \mathrm{~g} / \mathrm{dL}$ ), angina or other high-risk conditions were also excluded. Both treatment groups were observed for more than one year.

\section{Study design}

A total of 102 patients with chronic hepatitis C participated in the study (Fig. 1). The patients treated with phlebotomy gave their consent to undergo phlebotomy after receiving an explanation of the benefits and potential risks of the procedure. The non-phlebotomized patients included those from whom consent for phlebotomy was not obtained.

Of the 102 participants, 50 were treated with glycyrrhizin injection and oral UDCA therapy without phlebotomy, including 40 patients whose baseline level of serum ferritin was within the normal range (normal-ferritin subgroup) and 10 patients whose ferritin level was above the normal range (high-ferritin subgroup). In the other treatment group, 52 patients were treated with glycyrrhizin injection and oral UDCA therapy with phlebotomy, including 31 patients in the normal-ferritin subgroup and 21 patients in the highferritin subgroup. During the study follow-up period, it was found that six of the 52 phlebotomized patients had received IFN therapy more than 10 years previously; therefore, the data for these six patients were excluded from the data analysis (Fig. 1).

The primary endpoints were the serum levels of AST, 
ALT, total bilirubin, alkaline phosphatase, gamma-glutamyl transpeptidase (G-GTP), lactate dehydrogenase (LDH) and albumin, as well as the hemoglobin concentration, platelet count, prothrombin time and incidence of HCC. In addition, the serum levels of alpha-fetoprotein (AFP), used as a tumor marker, were determined using an enzyme immunoassay.

\section{Measurement of the serum ferritin and iron concen- trations}

We also determined the serum ferritin and iron concentrations. The serum ferritin concentrations were evaluated using a chemiluminescent enzyme immunoassay (Lumispot Eiken Ferritin; Eiken Chemical Co., Ltd., Tokyo, Japan), while the serum iron concentrations were evaluated according to the Nitroso-PSAP method (Quickauto-Neo Fe; Shino-Test Corp., Tokyo, Japan).

\section{Definitive diagnosis of HCC}

All patients were assessed two or three times each year to check for space-occupying lesions based on the findings of abdominal ultrasonography and computed tomography examinations, and definitive diagnoses of HCC were made using abdominal angiography performed between January 2005 and December 2012.

\section{Method for phlebotomy}

Depending on the individual patient's condition and blood pressure, phlebotomy was performed to remove $100-400 \mathrm{~mL}$ of blood every 1-4 weeks. If the patient's serum hemoglobin level was below $10 \mathrm{~g} / \mathrm{dL}$, phlebotomy was not performed. After phlebotomy, the serum transaminases, ferritin and hemoglobin levels were tested monthly. The method used to remove blood was tightly controlled and included using a syringe for injection and a blood donation bag.

\section{Dietary and nutritional instructions}

Registered dietitians were instructed to design a low-iron diet that included only $6 \mathrm{mg}$ of iron per day. We explained to the patients that a limited oral intake of iron can improve the liver function and suppress the progression of liver disease. The patients were asked not to use tableware containing iron and were provided a chart showing the constituents of foods.

\section{Statistical analysis}

Differences between the treatment groups and the highversus normal-ferritin subgroups were analyzed using the unpaired t-test and an analysis of variance. The chi-square test, Mann-Whitney U-test, multivariate analysis of variance and the Kaplan-Meier method were used to compare the incidence of HCC based on various factors, including the treatment group and baseline laboratory values. The between-group differences in the carcinogenesis curves were assessed using the log-rank test. A p-value of $<0.05$ was considered to be statistically significant. All data analyses were performed using the computer software program SPSS version 11 (SPSS Inc., Chicago, USA).

\section{Results}

\section{Patient characteristics in the treatment groups}

The baseline characteristics of the study patients are summarized in Table 1. Comparing the phlebotomy group versus the non-phlebotomy group, the serum AST $(88 \pm 50$ vs. $57 \pm$ $36 \mathrm{IU} / \mathrm{L}, \mathrm{p}<0.01)$ and ALT $(108 \pm 49$ vs. $59 \pm 40$ IU/L, $\mathrm{p}<$ $0.01)$ levels were lower in the non-phlebotomy group. However, the mean ferritin concentration at baseline was significantly higher in the phlebotomy group $(270 \pm 232 \mu \mathrm{g} / \mathrm{L})$ than in the non-phlebotomy group $(139 \pm 172 \mu \mathrm{g} / \mathrm{L}, \mathrm{p}<0.01)$. There were no statistically significant baseline differences in the platelet count, total bilirubin level, prothrombin time or serum albumin level.

\section{Changes in the serum laboratory values according to the treatment group}

After the initiation of phlebotomy, the serum ferritin levels immediately exhibited a gradual decline. In the phlebotomy group, the mean ferritin level $( \pm \mathrm{SD})$ was $270 \pm 232 \mu \mathrm{g} /$ $\mathrm{L}$ at baseline $(\mathrm{n}=46)$ and $63 \pm 81 \mu \mathrm{g} / \mathrm{L}$ after five years $(\mathrm{n}=$ 30) (Fig. 2).

In contrast, no substantial changes were observed in the group that did not undergo phlebotomy. In the nonphlebotomy group, the mean ferritin level $( \pm$ SD) was $139 \pm$ $172 \mu \mathrm{g} / \mathrm{L}$ at baseline $(\mathrm{n}=50)$ and $74 \pm 72 \mu \mathrm{g} / \mathrm{L}$ after five years $(n=30)$ (Fig. 2). The ferritin level not be controlled in only five patients, as these phlebotomized patients did not adhere to the low-iron diet.

The mean serum AST level in the phlebotomy group was decreased at each one-year interval for five years after the phlebotomy treatment (e.g. from $88 \pm 50 \mathrm{IU} / \mathrm{L}$ at baseline to $48 \pm 24 \mathrm{IU} / \mathrm{L}$ at 5 years). However, the AST levels remained unchanged in the non-phlebotomy group at most of the oneyear intervals over the five-year period (e.g. from $57 \pm 36$ IU/ $\mathrm{L}$ at baseline to $46 \pm 20 \mathrm{IU} / \mathrm{L}$ at 5 years). There were significant differences between the two groups $(\mathrm{p}<0.04)$ (Fig. 2). The mean serum ALT level in the phlebotomy group was decreased at each one-year interval for five years after the phlebotomy treatment (e.g. from $108 \pm 49$ IU/L at baseline to $50 \pm 29 \mathrm{IU} / \mathrm{L}$ at 5 years), whereas the ALT levels remained unchanged in the non-phlebotomy group at most of the oneyear intervals over the five-year period (e.g. from $59 \pm 40 \mathrm{IU} /$ $\mathrm{L}$ at baseline to $46 \pm 20 \mathrm{IU} / \mathrm{L}$ at 5 years). There were significant differences between the two groups $(\mathrm{p}<0.01)$ (Fig. 2). The mean platelet count in the phlebotomy group was not decreased at most of the one-year intervals during the fiveyear period (e.g. from $16.1 \pm 5.1 \times 10^{4} / \mathrm{mm}^{3}$ at baseline to $15.8 \pm 5.4 \times 10^{4} / \mathrm{mm}^{3}$ at 5 years), while the platelet counts in the non-phlebotomy group were decreased at most of the one-year intervals for five years (e.g. from $18.2 \pm 8.0 \times 10^{4}$ / $\mathrm{mm}^{3}$ at baseline to $\left.16.7 \pm 6.7 \times 10^{4} / \mathrm{mm}^{3}\right)$. There were no significant differences between the two groups $(p=0.73)$ 
Table 1. Baseline Characteristics of the Patients with Chronic Hepatitis $\mathrm{C}$ between the Phlebotomy and Non-phlebotomy Groups

\begin{tabular}{lccc}
\hline Variables & Phlebotomy group & Non-phlebotomy group & p value \\
\hline Patients $(\mathrm{n})$ & 46 & 50 & \\
Age (years) & $55 \pm 12$ & $61 \pm 14$ & 0.11 \\
Gender (male: female) & $30: 16$ & $22: 28$ & 0.26 \\
Hemoglobin $(\mathrm{g} / \mathrm{dL})$ & $14.7 \pm 1.1$ & $13.8 \pm 1.7$ & 0.44 \\
Platelet count $\left(\times 10^{4} / \mathrm{mm}^{3}\right)$ & $15.8 \pm 4.9$ & $18.2 \pm 8.0$ & 0.09 \\
AST $(\mathrm{IU} / \mathrm{L})$ & $88 \pm 50$ & $57 \pm 36$ & $<0.01$ \\
ALT $(\mathrm{IU} / \mathrm{L})$ & $108 \pm 49$ & $59 \pm 40$ & $<0.01$ \\
G-GTP $(\mathrm{IU} / \mathrm{L})$ & $75 \pm 89$ & $52 \pm 68$ & 0.15 \\
LDH $(\mathrm{IU} / \mathrm{L})$ & $206 \pm 54$ & $179 \pm 52$ & 0.86 \\
Total bilirubin $(\mathrm{mg} / \mathrm{dL})$ & $0.7 \pm 0.25$ & $0.7 \pm 0.5$ & 0.98 \\
Alkaline phosphatase $(\mathrm{IU} / \mathrm{L})$ & $323 \pm 124$ & $317 \pm 107$ & 0.65 \\
Serum albumin $(\mathrm{g} / \mathrm{dL})$ & $4.0 \pm 0.3$ & $3.8 \pm 0.5$ & 0.23 \\
Prothrombin time $(\%)$ & $95 \pm 9$ & $97 \pm 6$ & 0.37 \\
Iron $(\mu \mathrm{g} / \mathrm{dL})$ & $204 \pm 255$ & $108 \pm 63$ & 0.17 \\
Ferritin $(\mu \mathrm{g} / \mathrm{L})$ & $270 \pm 232$ & $139 \pm 172$ & $<0.01$ \\
AFP $(\mathrm{ng} / \mathrm{mL})$ & $8.8 \pm 13.0$ & $8.8 \pm 12.7$ & 0.85 \\
\hline
\end{tabular}

The values presented are primarily the mean \pm SD.

AFP: alpha-fetoprotein, ALT: alanine aminotransferase, AST: aspartate aminotransferase, G-GTP: gamma-glutamyl transpeptidase, LDH: lactate dehydrogenase, SD: standard deviation
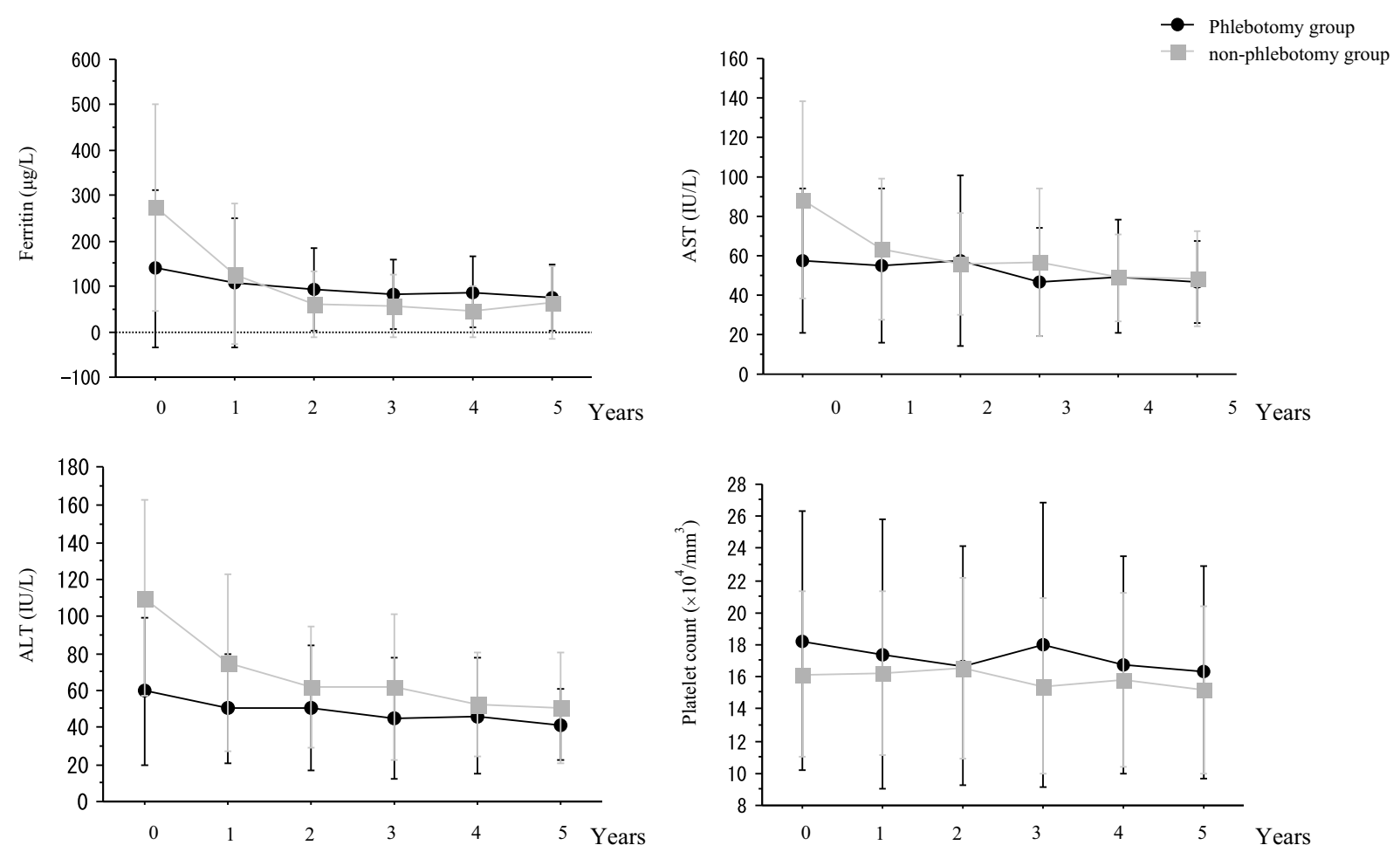

Figure 2. Comparison of the mean serum ferritin, AST and ALT levels and platelet count between the phlebotomy and non-phlebotomy groups. Six patients in the phlebotomy group were excluded from the data analysis due to a history of previous IFN therapy.

(Fig. 2). In addition, the alkaline phosphatase, G-GTP and albumin levels and prothrombin time showed no significant differences between the groups.

Among the five phlebotomized patients whose ferritin level could not be controlled, the mean AST, ALT and plate- let levels did not change significantly during the five-year study period, although the transaminase levels decreased (i. e. AST from $127 \pm 68 \mathrm{IU} / \mathrm{L}$ at baseline to $69 \pm 32 \mathrm{IU} / \mathrm{L}$ at 5 years, $\mathrm{p}=0.57$; ALT from $135 \pm 64$ IU/L to $48 \pm 44 \mathrm{IU} / \mathrm{L}, \mathrm{p}=$ 0.11 ; and platelet count from $12.2 \pm 3.8 \times 10^{4} / \mathrm{mm}^{3}$ to $14.1 \pm$ 

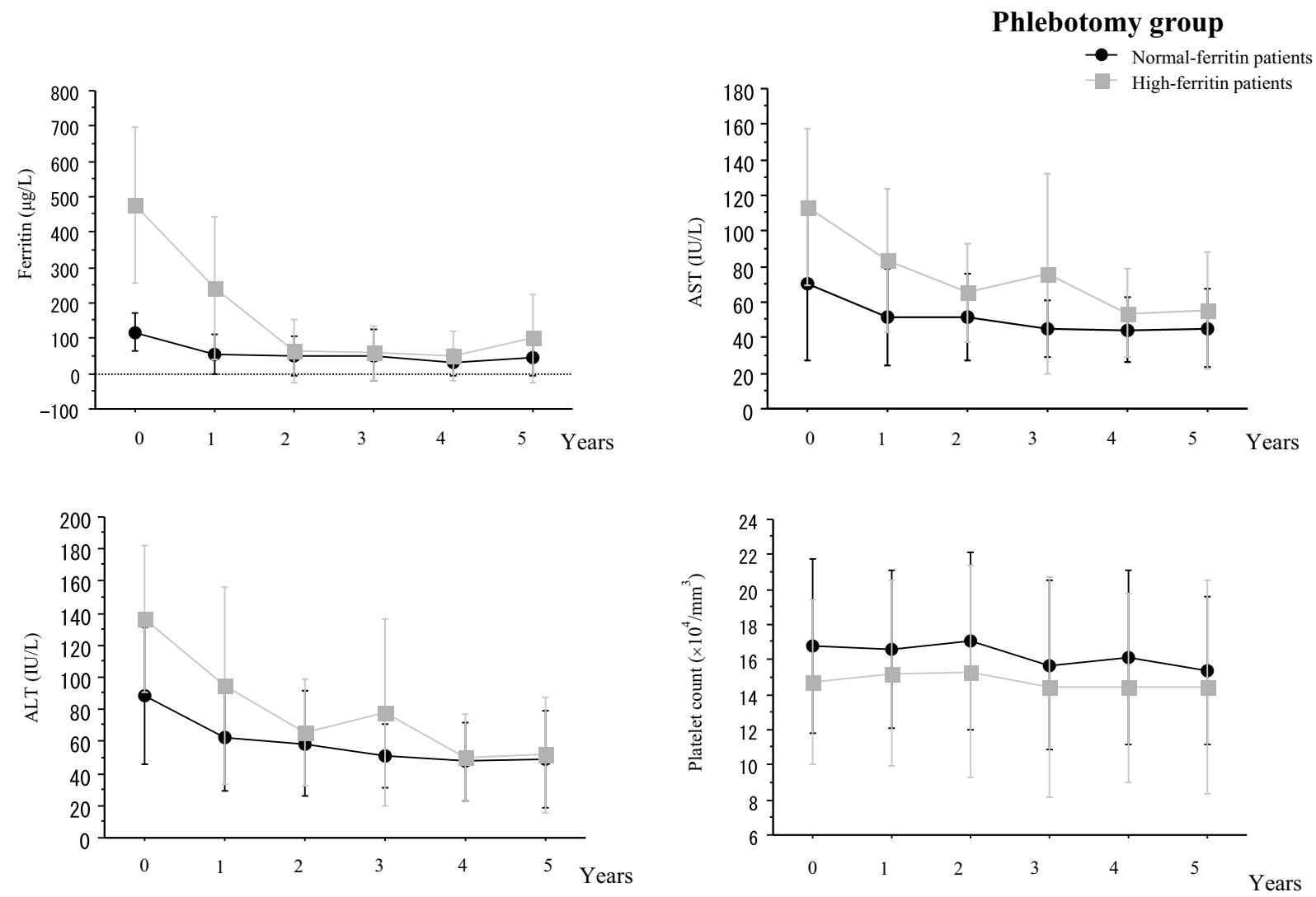

Figure 3. Comparison of the mean serum ferritin, AST and ALT levels and platelet count between the patients with normal versus high baseline ferritin levels in the phlebotomy group.

$\left.5.5 \times 10^{4} / \mathrm{mm}^{3}, \mathrm{p}=0.16\right)$.

\section{AFP levels at baseline}

In terms of the serum levels of AFP, the mean baseline AFP level was not significantly different between the phlebotomy and non-phlebotomy groups (Table 1).

\section{Changes in the serum laboratory values according to the baseline ferritin level}

We compared the changes in the serum laboratory values (ferritin, ALT, AST, and platelet count) in the phlebotomized patients with normal versus high baseline ferritin levels (Fig. 3)

The serum ferritin levels immediately exhibited a gradual decline after phlebotomy. Among the phlebotomized patients with a normal baseline ferritin level, the mean ferritin value $( \pm \mathrm{SD})$ was $116 \pm 53 \mu \mathrm{g} / \mathrm{L}$ at baseline $(\mathrm{n}=27)$ and $43 \pm 50 \mu \mathrm{g} / \mathrm{L}$ after five years $(\mathrm{n}=20)$ (Fig. 3). Among those with a high baseline ferritin level, the mean ferritin value $( \pm \mathrm{SD})$ was $476 \pm 218 \mu \mathrm{g} / \mathrm{L}$ at baseline $(\mathrm{n}=19)$ and $102 \pm 117 \mu \mathrm{g} / \mathrm{L}$ after five years $(n=10)$. There were significant differences within and between the two subgroups ( $\mathrm{p}<0.01)$ (Fig. 3).

The mean serum AST level in the phlebotomized patients with a normal baseline ferritin level was significantly decreased at each one-year interval for five years after the phlebotomy treatment (e.g. from $70 \pm 42 \mathrm{IU} / \mathrm{L}$ at baseline to $45 \pm 22 \mathrm{IU} / \mathrm{L}$ at 5 years). The AST levels were also decreased in the high-ferritin patients at most of the one-year intervals for five years (e.g. from $113 \pm 44 \mathrm{IU} / \mathrm{L}$ at baseline to $55 \pm 32$ $\mathrm{IU} / \mathrm{L}$ at 5 years). There were significant differences within and between the two subgroups ( $\mathrm{p}=0.02$ ) (Fig. 3). The mean serum ALT level in the phlebotomized patients with a normal baseline ferritin level was significantly decreased at each one-year interval for five years after the phlebotomy treatment (e.g. from $88 \pm 42 \mathrm{IU} / \mathrm{L}$ at baseline to $48 \pm 30 \mathrm{IU} / \mathrm{L}$ at 5 years). The ALT levels were also decreased in the highferritin patients at most of the one-year intervals for five years (e.g. from 136 at baseline $\pm 46 \mathrm{IU} / \mathrm{L}$ to $51 \pm 36 \mathrm{IU} / \mathrm{L}$ at 5 years). There were significant differences within and between the two subgroups ( $\mathrm{p}=0.04)$ (Fig. 3). The mean platelet count in the phlebotomized patients with a normal or high baseline ferritin level was not significantly decreased at any of the one-year intervals during the five-year study period (e.g. from $16.7 \pm 4.9 \times 10^{4} / \mathrm{mm}^{3}$ at baseline to $15.3 \pm 4.2 \times$ $10^{4} / \mathrm{mm}^{3}$ at 5 years in patients with normal ferritin; and from $14.7 \pm 4.7 \times 10^{4} / \mathrm{mm}^{3}$ at baseline to $14.4 \pm 6.1 \times 10^{4} / \mathrm{mm}^{3}$ at 5 years in those with high ferritin). There were no significant differences within or between the two subgroups $(\mathrm{p}=0.35)$ (Fig. 3).

\section{Effects of phlebotomy on the incidence of HCC}

Of the 96 study patients, 19 were diagnosed with HCC during the follow-up period. HCC developed in four $(10.3 \%)$ patients in the phlebotomy group and 15 (30.0\%) patients in the non-phlebotomy group.

Based on the number of remaining study patients at risk 


\section{All patients:}

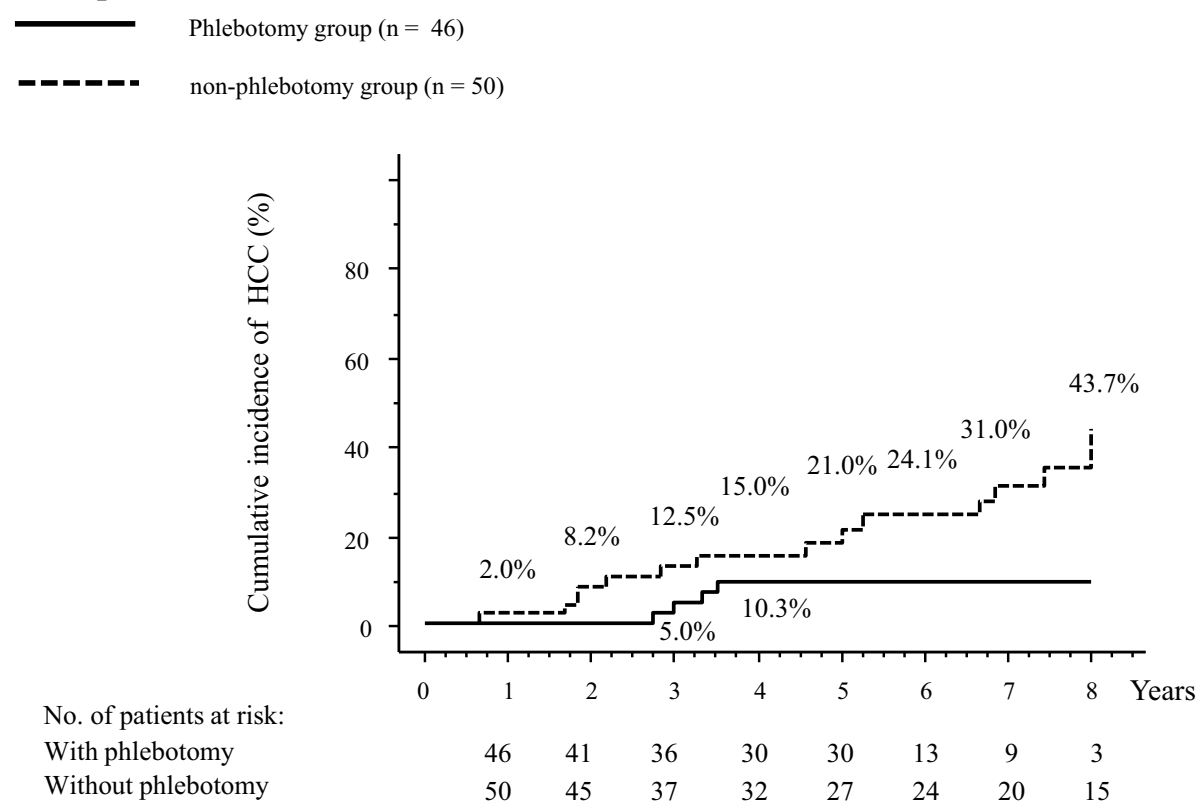

Figure 4. Comparison of the cumulative incidence of HCC between the phlebotomy and non-phlebotomy groups: all patients. The log-rank test revealed a statistically significant between-group difference in the cumulative incidence of $\mathrm{HCC}$ at eight years $(\mathrm{p}=0.04)$.

Table 2. Multivariate Analysis of the Impact of the Variables on the Incidence of HCC

\begin{tabular}{lccc}
\hline Variables & Risk ratio & 95\% Confidence interval & $\mathrm{p}$ value \\
\hline Phlebotomy & 0.13 & $0.26-0.67$ & 0.01 \\
Age & 1.03 & $0.96-1.01$ & 0.33 \\
Platelet count & 0.94 & $0.84-1.05$ & 0.27 \\
AST & 1.02 & $0.09-1.05$ & 0.08 \\
ALT & 0.98 & $0.96-1.01$ & 0.4 \\
AFP & 1.01 & $0.98-1.03$ & 0.39 \\
\hline
\end{tabular}

A multivariate analysis was performed to determine the impact of phlebotomy and other variables on the incidence of HCC. Factors such as phlebotomy, age, platelet count and the AST and AFP levels were assessed using a logistic regression analysis. The only statistically significant difference in the risk of HCC was observed between the phlebotomy and non-phlebotomy groups.

over time, the cumulative incidence of $\mathrm{HCC}$ for individual years in the phlebotomy group was $0.0 \%$ at years $1-2,5.0 \%$ at year 3 and $10.3 \%$ at years $4-8$. The incidence of HCC in the non-phlebotomy group was $2.0 \%$ at year $1,8.2 \%$ at year $2,12.5 \%$ at year $3,15.0 \%$ at year $4,21.0 \%$ at year 5 , $24.1 \%$ at year $6,31.0 \%$ at year 7 and $43.7 \%$ at year 8 . The log-rank test revealed a statistically significant difference in the incidence of HCC between the phlebotomy group and the non-phlebotomy group over time $(\mathrm{p}=0.04)$, with a lower risk associated with phlebotomy (Fig. 4).

During follow-up, HCC was diagnosed in one of the five patients with an uncontrollable ferritin level in the phlebotomy group.

\section{Multivariate analysis of the factorial impact on the incidence of HCC}

The Mann-Whitney U-test and chi-square test revealed statistically significant differences indicating that phlebotomy $(\mathrm{p}<0.01)$, age $(\mathrm{p}<0.01)$ and the baseline platelet count $(\mathrm{p}=0.01)$ and AST $(\mathrm{p}=0.04)$, ALT $(\mathrm{p}=0.01)$ and AFP $(\mathrm{p}<$ 0.01 ) levels had substantial effects on the incidence of HCC.

Factors such as phlebotomy, age and the baseline platelet count and AST and AFP levels were also assessed using a logistic regression analysis to determine their impact on the incidence of HCC (Table 2). The only statistically significant difference in risk of HCC was between the phlebotomy and non-phlebotomy groups, with phlebotomy offering a protective risk ratio of 0.13 .

\section{Baseline characteristics of the patients with a nor- mal baseline ferritin level}

The characteristics of the patients with a normal baseline serum ferritin level are summarized in Table 3. At baseline, the mean hemoglobin level was significantly lower in the non-phlebotomy group $(15.0 \pm 1.1$ vs. $13.8 \pm 4.2 \mathrm{~g} / \mathrm{dL}, \mathrm{p}<$ $0.01)$. The serum AST levels were not significantly different in the non-phlebotomy group $(70 \pm 42$ vs. $56 \pm 36 \mathrm{IU} / \mathrm{L}, \mathrm{p}=$ $0.15)$; however, the ALT levels ( $88 \pm 42$ vs. $56 \pm 38 \mathrm{IU} / \mathrm{L}, \mathrm{p}<$ $0.01)$ were significantly lower in the non-phlebotomy group. There were no significant between-group differences in the platelet count, prothrombin time or serum levels of AFP, GGTP, LDH, alkaline phosphatase, total bilirubin or albumin. 
Table 3. Baseline Characteristics of the Normal-ferritin Patients with Chronic Hepatitis $\mathrm{C}$ between the Phlebotomy and Non-phlebotomy Groups

\begin{tabular}{lccc}
\hline Variables & Phlebotomy group & Non-phlebotomy group & p value \\
\hline Patients (n) & 27 & 40 & \\
Age (years) & $52 \pm 7$ & $57 \pm 15$ & 0.08 \\
Gender (male: female) & $17: 10$ & $20: 20$ & 0.42 \\
Hemoglobin (g/dL) & $15.0 \pm 1.1$ & $13.8 \pm 4.2$ & $<0.01$ \\
Platelet count $\left(\times 10^{4} / \mathrm{mm}^{3}\right)$ & $16.9 \pm 4.9$ & $18.2 \pm 8.7$ & 0.40 \\
AST (IU/L) & $70 \pm 42$ & $56 \pm 36$ & 0.15 \\
ALT (IU/L) & $88 \pm 42$ & $56 \pm 38$ & $<0.01$ \\
G-GTP (IU/L) & $66 \pm 106$ & $55 \pm 80$ & 0.69 \\
LDH (IU/L) & $195 \pm 40$ & $178 \pm 54$ & 0.25 \\
Total bilirubin (mg/dL) & $0.7 \pm 0.2$ & $0.7 \pm 0.5$ & 0.85 \\
Alkaline phosphatase (IU/L) & $284 \pm 66$ & $312 \pm 111$ & 0.24 \\
Serum albumin (g/dL) & $4.1 \pm 0.3$ & $3.8 \pm 0.4$ & 0.28 \\
Prothrombin time (\%) & $98 \pm 3$ & $97 \pm 7$ & 0.39 \\
AFP (ng/mL) & $5 \pm 5$ & $9 \pm 14$ & 0.17 \\
\hline
\end{tabular}

The values presented are primarily the Mean \pm SD.

AFP: alpha-fetoprotein, ALT: alanine aminotransferase, AST: aspartate aminotransferase, G-GTP: gamma-glutamyl transpeptidase, LDH: lactate dehydrogenase, SD: standard deviation

Table 4. Baseline Characteristics of the High-ferritin Patients with Chronic Hepatitis C between the Phlebotomy and Non-phlebotomy Groups

\begin{tabular}{lccc}
\hline Variables & Phlebotomy group & Non-phlebotomy group & p value \\
\hline Patients (n) & 19 & 10 & \\
Age (years) & $60 \pm 10$ & $64 \pm 7$ & 0.31 \\
Gender (male: female) & $12: 7$ & $6: 4$ & 0.71 \\
Hemoglobin (g/dL) & $14.7 \pm 1.1$ & $13.8 \pm 1.5$ & 0.04 \\
Platelet count $\left(\times 10^{4} / \mathrm{mm}^{3}\right)$ & $15.8 \pm 4.9$ & $17.1 \pm 5.2$ & 0.47 \\
AST (IU/L) & $88 \pm 47$ & $63 \pm 40$ & 0.13 \\
ALT (IU/L) & $108 \pm 49$ & $70 \pm 46$ & 0.03 \\
G-GTP (IU/L) & $85 \pm 58$ & $53 \pm 29$ & 0.47 \\
LDH (IU/L) & $219 \pm 68$ & $198 \pm 52$ & 0.66 \\
Total bilirubin (mg/dL) & $0.7 \pm 0.6$ & $0.6 \pm 0.2$ & 0.36 \\
Alkaline phosphatase (IU/L) & $323 \pm 124$ & $313 \pm 96$ & 0.81 \\
Serum albumin (g/dL) & $4.0 \pm 0.3$ & $3.6 \pm 7.5$ & 0.78 \\
Prothrombin time (\%) & $92 \pm 12$ & $100 \pm 0$ & 0.31 \\
AFP (ng/mL) & $8.8 \pm 13.9$ & $7 \pm 4$ & 0.84 \\
\hline
\end{tabular}

The values presented are primarily the mean \pm SD.

AFP: alpha-fetoprotein, ALT: alanine aminotransferase, AST: aspartate aminotransferase, G-GTP: gamma-glutamyl transpeptidase, LDH: lactate dehydrogenase, SD: standard deviation

\section{Baseline characteristics of the patients with a high baseline ferritin level}

The characteristics of the patients with a high baseline serum ferritin level are summarized in Table 4. At baseline, the mean hemoglobin $(14.7 \pm 1.1$ vs. $13.8 \pm 1.5 \mathrm{~g} / \mathrm{dL}$, respectively, $\mathrm{p}=0.04)$ and ALT $(108 \pm 49$ vs. $70 \pm 46 \mathrm{IU} / \mathrm{L}, \mathrm{p}=0.03)$ levels were significantly lower in the non-phlebotomy group. There were no significant between-group differences in the platelet count, prothrombin time or serum levels of AST, AFP, G-GTP, LDH, alkaline phosphatase, total bilirubin or albumin.

\section{Incidence of HCC in the patients with a normal baseline ferritin level}

In the phlebotomy group, no patients with a normal serum ferritin level at baseline developed HCC during the study. In the non-phlebotomy group, the cumulative inci- 


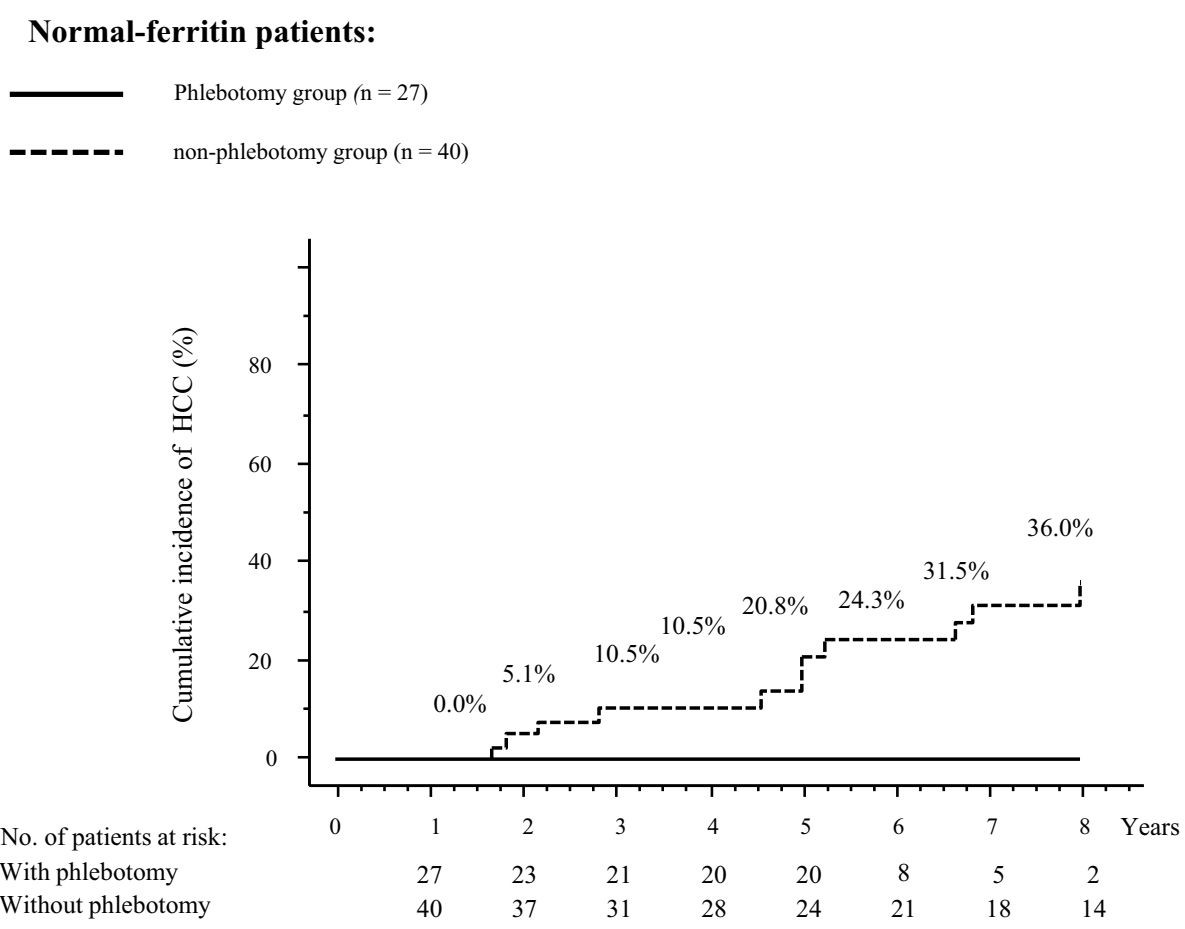

Figure 5. Comparison of the cumulative incidence of HCC between the phlebotomy and non-phlebotomy groups: patients with a normal baseline ferritin level. In the phlebotomy group, no normalferritin patients developed HCC. The cumulative incidence of $\mathrm{HCC}$ in the non-phlebotomy group was $36.0 \%$ at year 8 .

dence of HCC among the normal-ferritin patients was $0.0 \%$ at year $1,5.1 \%$ at year $2,10.5 \%$ at years $3-4,20.8 \%$ at year $5,24.3 \%$ at year $6,31.5 \%$ at year 7 and $36.0 \%$ at year 8 (Fig. 5).

\section{Incidence of HCC in the patients with a high base- line ferritin level}

Among the patients with a high baseline serum ferritin level, the cumulative incidence of $\mathrm{HCC}$ in the phlebotomy group was $0.0 \%$ at years $1-2,13.3 \%$ at year 3 and $27.8 \%$ at years 4-8. Meanwhile, the incidence of HCC in the nonphlebotomy group was $10.0 \%$ at year $1,20.0 \%$ at years $2-3$, $36.0 \%$ at years $4-7$ and $100 \%$ at year 8 . At eight years, the log-rank test showed no statistically significant betweengroup differences $(\mathrm{p}=0.15)$; however, the phlebotomy group exhibited a consistent trend of a numerically lower incidence of HCC, which suggested a lower risk (Fig. 6).

\section{Adverse events}

Complaints of feeling cold in the extremities prompted phlebotomy to be stopped in one patient. No significant adverse events occurred in other patients, and no patients discontinued therapy as a result of adverse events.

\section{Discussion}

The results of the present study suggest that long-term phlebotomy significantly improves the levels of transaminases and suppresses the development of HCC in HCV-
RNA-positive patients. Phlebotomy was shown to be especially beneficial in patients with a normal serum ferritin level. These results support the use of phlebotomy in patients with chronic hepatitis $\mathrm{C}$ who cannot receive or show no response to antiviral therapy.

In previous studies, patients with chronic hepatitis C exhibited significantly improved ALT levels following phlebotomy $(15,16)$, which is also associated with histological improvement (17). In a study of combined phlebotomy and UDCA treatment in patients with chronic hepatitis $\mathrm{C}$ who were only partial responders to monotherapy, the ALT levels improved to the upper normal range, with no statistically significant differences between either sequence of the combined treatments (18). Another study demonstrated that iron reduction therapy significantly improved the serum levels of aminotransferases and AFP in patients with HCV-related liver cirrhosis who had received UDCA and/or glycyrrhizin injection for more than six months before study entry (19). Similarly, the addition of petit phlebotomy was found to be effective and safe for improving the serum aminotransferase levels in hepatitis $\mathrm{C}$ patients receiving regular glycyrrhizin injections (20).

Our study results demonstrated that the levels of aminotransferases were significantly improved in the phlebotomy-treated patients, similar to the reported findings of earlier studies (18-20). In the present study, the significant improvements in the ALT and AST levels in the phlebotomy group suggest that the effects of phlebotomy augment the benefits of other therapies (Fig. 2). In addition, as 


\section{High-ferritin patients:}

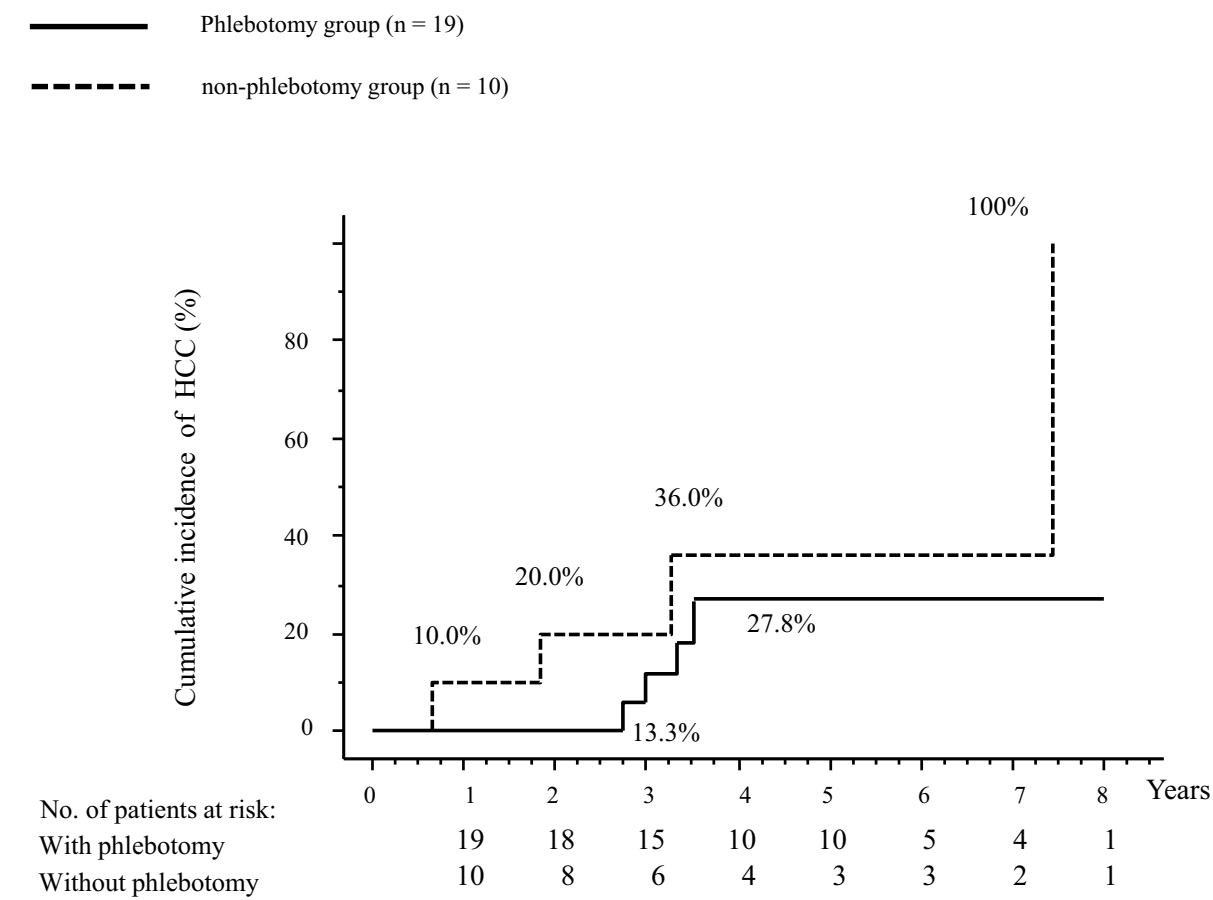

Figure 6. Comparison of the cumulative incidence of $\mathrm{HCC}$ between the phlebotomy and non-phlebotomy groups: patients with a high baseline ferritin level. The cumulative incidence of HCC among the high-ferritin patients in the phlebotomy group was $27.8 \%$ at years $4-8$. The cumulative incidence of HCC among the high-ferritin patients in the non-phlebotomy group was $36.0 \%$ at years $4-7$ and $100 \%$ at year 8 . The log-rank test revealed no statistically significant between-group differences in the cumulative incidence of $\mathrm{HCC}$ at eight years $(\mathrm{p}=0.15)$.

shown in Fig. 3, AST and ALT improvement was particularly apparent in the high-ferritin patients in the phlebotomy group.

It has been shown that $\mathrm{HCV}$-positive patients with mutations in the hemochromatosis gene (21-23), $\beta$-globin gene $(24,25)$ or hepcidin gene promoter $(25)$ have higher iron levels in the liver, along with more severe fibrosis and cirrhosis. In addition, the beneficial effects of iron depletion on serum markers of fibrogenesis in patients with chronic hepatitis $\mathrm{C}$ have been previously reported, with a significant reduction in the level of serum procollagen III peptide (26).

We initially predicted an improvement in the functional reserve of the liver; however, the prothrombin times and albumin levels were not significantly different between the two treatment groups. In addition, the platelet counts were not significantly decreased in the phlebotomy group at most of the one-year intervals over the first five years of the eight-year study period (Fig. 2).

In a clinical trial of phlebotomy for non-alcoholic fatty liver disease, the AST and ALT levels were found to be improved by phlebotomy (27). However, there were no improvements in histological lobular inflammation, steatosis, hepatocyte ballooning or fibrosis. These findings are consistent with the results for our patients, in whom phlebotomy improved the liver function but not the functional reserve of the liver.

It has also been reported that the rate of HCC develop- ment can be decreased by phlebotomy (13). Therefore, in this study, we also examined the incidence of HCC in patients treated with or without phlebotomy. During the observation period, the incidence of HCC was four of 46 patients $(10.3 \%)$ in the phlebotomy group and 15 of 50 patients $(30.0 \%)$ in the non-phlebotomy group, a statistically significant difference $(\mathrm{p}=0.04)$. The multivariate analysis also revealed statistically significant differences in the incidence of HCC between the phlebotomy and non-phlebotomy groups, with phlebotomy offering a protective risk ratio of 0.13 . These results show that the incidence of HCC can be improved by phlebotomy treatment.

Hereditary hemochromatosis is a well-known disease characterized by iron overload. Patients with this condition reportedly have high rates of HCC, cardiomyopathy, diabetes mellitus and extrahepatic carcinoma $(28,29)$. However, phlebotomy has been shown to improve the rate of carcinogenesis and prognosis of hereditary hemochromatosis $(29,30)$. In patients with hepatitis $\mathrm{C}$, it is thought that $\mathrm{HCV}$ is the cause of hepatic and extrahepatic disease, including HCC and non-Hodgkin's lymphoma (31-34). Oxidative stress is also considered to be a cause of HCVassociated HCC $(35,36)$. This theory is supported by the finding that iron reduction therapy with phlebotomy reduces lipid peroxidation and oxidative stress in patients with chronic hepatitis $\mathrm{C}$ (37).

We studied the effects of phlebotomy in patients with 
chronic hepatitis $\mathrm{C}$ and a high baseline ferritin level. In the phlebotomized patients with a high ferritin level, the cumulative incidence of HCC was $27.8 \%$ at years 4-8 (Fig. 6). In comparison, in the non-phlebotomy patients with a high ferritin level, the cumulative incidence of $\mathrm{HCC}$ was $36.0 \%$ at years $4-7$ and $100 \%$ at year 8 . Among the patients with a high baseline ferritin level, there were no statistically significant differences in the cumulative incidence of HCC between the phlebotomy and non-phlebotomy groups at eight years. However, the cumulative incidence of HCC tended to be lower in the high-ferritin patients in the phlebotomy group. We postulated that this lack of a significant difference was due to the small number of patients remaining in the study at eight years. Another consideration is the background characteristics of the high-ferritin patients, in particular, the significantly higher baseline levels of ALT in the patients treated with phlebotomy (indicating more advanced liver disease) than in the non-phlebotomy group ( $\mathrm{p}=0.03$, Table 4). However, despite the presence of more advanced disease in the phlebotomized patients, the cumulative incidence of HCC was not significantly different compared to that observed in the non-phlebotomy group. We therefore suggest that phlebotomy can be used to suppress the incidence of HCC, even in high-ferritin patients.

The present study's perhaps most impressive finding is that, among the patients with a normal baseline ferritin level, no phlebotomized patients developed HCC, while the incidence of HCC in the matched subgroup treated without phlebotomy was $36 \%$ at year 8 (Fig. 5). We concluded that these data showed that phlebotomy is more effective at suppressing the risk of $\mathrm{HCC}$ in $\mathrm{HCV}$-infected patients with a normal iron level. We recommend that patients with chronic hepatitis $\mathrm{C}$ receive phlebotomy before iron begins to accumulate.

The authors state that they have no Conflict of Interest (COI).

\section{Acknowledgement}

The investigators who participated in this study are as follows:

Y. Arakawa (Nihon University), A. Higuchi (Nihon University), N. Tanaka (Nihon University) and H. Yamagami (Nihon University).

\section{References}

1. McHutchison JG, Everson GT, Gordon SC, et al. Telaprevir with peginterferon and ribavirin for chronic $\mathrm{HCV}$ genotype 1 infection. N Engl J Med 360: 1827-1838, 2009.

2. McHutchison JG, Manns MP, Muir AJ, et al. Telaprevir for previously treated chronic HCV infection. N Engl J Med 362: 1292$1303,2010$.

3. Hézode C, Forestier N, Dusheiko G, et al. Telaprevir and peginterferon with or without ribavirin for chronic HCV infection. N Engl J Med 360: 1839-1850, 2009.

4. Akuta N, Suzuki F, Sezaki H, et al. Predictive factors of virological non-response to interferon-ribavirin combination therapy for patients infected with hepatitis $\mathrm{C}$ virus of genotype $1 \mathrm{~b}$ and high viral load. J Med Virol 78: 83-90, 2006.

5. Enomoto N, Sakuma I, Asahina Y, et al. Mutations in the non- structural protein $5 \mathrm{~A}$ gene and response to interferon in patients with chronic hepatitis $\mathrm{C}$ virus $1 \mathrm{~b}$ infection. $\mathrm{N}$ Engl $\mathrm{J}$ Med 334: 77-81, 1996.

6. Ge D, Fellay J, Thompson AJ, et al. Genetic variation in IL28B predicts hepatitis $C$ treatment-induced viral clearance. Nature 461 : 399-401, 2009.

7. Tanaka Y, Nishida N, Sugiyama M, et al. Genome-wide association of IL28B with response to pegylated interferon-alpha and ribavirin therapy for chronic hepatitis C. Nat Genet 41: 11051109, 2009.

8. Kumada H. Long-term treatment of chronic hepatitis $\mathrm{C}$ with glycyrrhizin [stronger neo-minophagen C (SNMC)] for preventing liver cirrhosis and hepatocellular carcinoma. Oncology 62: 94-100, 2002.

9. Ikeda K, Arase Y, Kobayashi M, et al. A long-term glycyrrhizin injection therapy reduces hepatocellular carcinogenesis rate in patients with interferon-resistant active chronic hepatitis C: a cohort study of 1249 patients. Dig Dis Sci 51: 603-609, 2006.

10. Rino Y, Tarao K, Morinaga S, et al. Reduction therapy of alanine aminotransferase levels prevent $\mathrm{HCC}$ development in patients with HCV-associated cirrhosis. Anticancer Res 26: 2221-2226, 2006.

11. Tarao K, Fujiyama S, Ohkawa S, et al. Ursodiol use is possibly associated with lower incidence of hepatocellular carcinoma in hepatitis C virus-associated liver cirrhosis. Cancer Epidemiol Biomarkers Prev 14: 164-169, 2005.

12. Abd Elmonem E, Tharwa S, Farag MA, et al. Hepcidin mRNA level as a parameter of disease progression in chronic hepatitis $\mathrm{C}$ and hepatocellular carcinoma. J Egypt Natl Canc Inst 21: 333-342, 2009.

13. Kato J, Miyanishi K, Kobune M, et al. Long-term phlebotomy with low-iron diet therapy lowers risk of development of hepatocellular carcinoma from chronic hepatitis C. J Gastroenterol 42: 830-836, 2007.

14. Adams PC, Reboussin DM, Barton JC, et al. Hemochromatosis and ironoverload screening in a racially diverse population. $\mathrm{N}$ Engl J Med 352: 1769-1778, 2005.

15. Hayashi H, Takikawa $T$, Nishimura $N$, Yano $M$, Isomura $T$, Sakamoto N. Improvement of serum aminotransferase levels after phlebotomy in patients with chronic active hepatitis $\mathrm{C}$ and excess hepatic iron. Am J Gastroenterol 89: 986-988, 1994.

16. Franchini M, Targher G, Capra F, Montagnana M, Lippi G. The effect of iron depletion on chronic hepatitis $\mathrm{C}$ virus infection. Hepatol Int 2: 335-340, 2008.

17. Sartori M, Andorno S, Rossini A, et al. Phlebotomy improves histology in chronic hepatitis $\mathrm{C}$ males with mild iron overload. World J Gastroenterol 16: 596-602, 2010.

18. Wakusawa S, Ikeda R, Takikawa T, Hayashi H, Yano M, Yoshioka K. Combined phlebotomy and ursodeoxycholic acid treatment in the patients with chronic hepatitis C. Hepatol Res 18: 54-62, 2000.

19. Tanaka N, Horiuchi A, Yamaura T, Komatsu M, Tanaka E, Kiyosawa K. Efficacy and safety of 6-month iron reduction therapy in patients with hepatitis $\mathrm{C}$ virus-related cirrhosis: a pilot study. J Gastroenterol 42: 49-55, 2007.

20. Tanaka N, Horiuchi A, Yamaura T, et al. Efficacy and safety of addition of minor bloodletting (petit phlebotomy) in hepatitis $\mathrm{C}$ virus-infected patients receiving regular glycyrrhizin injections. J Gastroenterol 44: 577-582, 2009.

21. Bonkovsky HL, Troy N, McNeal K, et al. Iron and HFE or TfR1 mutations as comorbid factors for development and progression of chronic hepatitis C. J Hepatol 37: 848-854, 2002.

22. Tung BY, Emond MJ, Bronner MP, Raaka SD, Cotler SJ, Kowdley KV. Hepatitis C,iron status, and disease severity: relationship with HFE mutations. Gastroenterology 124: 318-326, 2003.

23. Erhardt A, Maschner-Olberg A, Mellenthin C, et al. HFE mutations and chronic hepatitis C: H63D andC282Y heterozygosity are 
independent risk factors for liver fibrosis and cirrhosis. J Hepatol 38: 335-342, 2003.

24. Sartori M, Andorno S, Pagliarulo M, et al. Heterozygous betaglobin gene mutations as a risk factor for iron accumulation and liver fibrosis in chronic hepatitis C. Gut 56: 693-698, 2007.

25. Valenti L, Pulixi EA, Arosio P, et al. Relative contribution of iron genes, dysmetabolism and hepatitis $\mathrm{C}$ virus (HCV) in the pathogenesis of altered iron regulation in HCV chronic hepatitis. Haematologica 92: 1037-1042, 2007.

26. Alexander J, Tung BY, Croghan A, Kowdley KV. Effect of iron depletion on serum markers of fibrogenesis, oxidative stress and serum liver enzymes in chronic hepatitis C: results of a pilot study. Liver Int 27: 268-273, 2007.

27. Beaton MD, Chakrabarti S, Levstik M, Speechley M, Marotta P, Adams P. Phase II clinical trial of phlebotomy for non-alcoholic fatty liver disease. Aliment Pharmacol Ther 37: 720-729, 2013.

28. Nelson RL, Davis FG, Persky V, Becker E. Risk of neoplastic and other diseases among people with heterozygosity for hereditary hemochromatosis. Cancer 76: 875-879, 1995.

29. Niederau C, Fischer R, Sonnenberg A, Stremmel W, Trampisch HJ, Strohmeyer G. Survival and causes of death in cirrhotic and in noncirrhotic patients with primary hemochromatosis. N Engl J Med 313: 1256-1262, 1985.

30. Niederau C, Fischer R, Pürschel A, Stremmel W, Häussinger D,
Strohmeyer G. Long-term survival in patients with hereditary hemochromatosis. Gastroenterology 110: 1107-1119, 1996.

31. Rosen HR, Gretch DR. Hepatitis C virus: Current understanding and prospects for future therapies. Mol Med Today 5: 393-399, 1999.

32. Hartridge-Lambert SK, Stein EM, Markowitz AJ, Portlock CS. Hepatitis $\mathrm{C}$ and non-Hodgkin lymphoma: the clinical perspective. Hepatology 55: 634-641, 2012.

33. Nocente R, Ceccanti M, Bertazzoni G, Cammarota G, Silveri NG, Gasbarrini G. HCV infection and extrahepatic manifestations. Hepatogastroenterology 50: 1149-1154, 2003.

34. Jin DY. Molecular pathogenesis of hepatitis $\mathrm{C}$ virus-associated hepatocellular carcinoma. Front Biosci 12: 222-233, 2007.

35. Tanaka N, Moriya K, Kiyosawa K, Koike K, Gonzalez FJ, Aoyama T. PPARalpha activation is essential for HCV core protein-induced hepatic steatosis and hepatocellular carcinoma in mice. J Clin Invest 118: 683-694, 2008.

36. Moriya K, Nakagawa K, Santa T, et al. Oxidative stress in the absence of inflammation in a mouse model for hepatitis $\mathrm{C}$ virusassociated hepatocarcinogenesis. Cancer Res 61: 4365-4370, 2001.

37. Kaito M, Iwasa M, Kobayashi Y, et al. Iron reduction therapy by phlebotomy reduces lipid peroxidation and oxidative stress in patients with chronic hepatitis C. J Gastroenterol 41: 921-922, 2006.

(C) 2015 The Japanese Society of Internal Medicine http://www.naika.or.jp/imonline/index.html 